\title{
BOUNDS ON THE NILPOTENCY CLASS OF CERTAIN SEMIDIRECT PRODUCTS
}

\author{
BY \\ LARRY MORLEY
}

\begin{abstract}
Gilbert Baumslag has shown that the standard wreath product of $A$ by $B$ is nilpotent if and only if $A$ and $B$ are $p$-groups for the same prime $p, A$ is nilpotent of bounded exponent and $B$ is finite. L. Kaloujnine and Marc Krasner have shown that the standard (unrestricted) wreath product of $A$ by $B$ contains an isomorphic copy of every group $G$ which is an extension of $A$ by $B$. Thus it follows that any extension subject to the above condition on $A$ and $B$ is nilpotent. In this paper, the author gives an explicit characterization of the terms of the lower central series of a semidirect product $W$ of an abelian group by an arbitrary group. He then establishes a formula for an upper bound on the nilpotency class of $W$ when $W$ is a semidirect product of an abelian $p$-group $X$ of bounded exponent by a finite $p$-group $B$. This new bound is given in terms of the exponent of $X$ and the cycle structure of the factor groups of the lower central series of $B$.
\end{abstract}

1. Introduction. From the results of Gilbert Baumslag [1] and L. Kaloujnine and Marc Krasner [3] it follows that a sufficient condition that an extension group $W$ of $X$ by $B$ be nilpotent is that $X$ and $B$ are $p$-groups for the same prime $p, X$ is nilpotent of bounded exponent, and $B$ is finite. It is the object of this paper to establish the best possible upper bound on the nilpotency class of the general semidirect product of $X$ by $B$ where $X$ is an abelian group of exponent $p^{n+1}$ and $B$ is a finite $p$-group. After the preliminaries of $\$ 2$ an explicit characterization of the lower central series of these semidirect products is formulated in $\S 3$. This characterization is then utilized in $\$ 4$ to establish the new upper bound on the nilpotency class. This upper bound generalizes the formula given by Liebeck [4] for the nilpotency class of the standard wreath product of two abelian $p$-groups and substantially improves the bound given by Scruton [7] for the more general case of the standard wreath product of two $p$-groups.

2. Preliminaries. If $g_{1}, \ldots, g_{n}$ are elements of a group $G$, then $\left(g_{1}, \ldots, g_{n}\right)$ denotes a commutator element of length $n$ in $G$. If $t$ is a positive integer, then $\left(g_{1}, t_{2}\right)$ denotes the commutator $\left(g_{1}, g_{2}, \ldots, g_{2}\right)$ with $g_{2}$ appearing as an entry $t$ times. The following commutator identities are well known.

(2.1) (i) $(x, y z)=(x, z)(x, y)(x, y, z)$,

(ii) $(x y, z)=(x, z)(x, z, y)(y, z)$.

Received by the editors September 25, 1970.

AMS 1970 subject classifications. Primary 20D15, 20E15, 20F25; Secondary 20F35.

Key words and phrases. Nilpotency class, lower central series, semidirect products, p-groups, wreath products.

Copyright (C) 1971, American Mathematical Society 
If $G$ is any group, then $Z_{i}(G), i=0,1,2, \ldots$, and $G_{(i)}, i=1,2,3, \ldots$, denote the upper and lower central series of $G$ respectively. An arbitrary ascending central series is denoted $\Lambda: G_{0}<G_{1}<G_{2}<\cdots$. For information on these and other normal series see Philip Hall [2].

If $W$ is an extension of $X$ by $B$, then $X$ is assumed to be a normal subgroup of $W$ and $W / X \cong B$. If $f$ and $t$ are specified elements, $f \in X$ and $t$ an element of a transversal $T$ of $X$ in $W$, then, following the simplified notation of Liebeck [4] for wreath products, $f_{i}=f^{t^{i}}$. In case $W$ is a semidirect product of $X$ by $B$, then $B$ is assumed to be a subgroup of $W$ and $T$ is replaced by $B$ itself.

The following lemma is a generalization of Liebeck [4, Lemma 3.1]. There is a slight correction in the sign of certain exponents, however this correction does not affect the divisibility properties discussed in Theorem 2.4. Theorem 2.4 follows from Liebeck [4, Theorem 4.3] and is a generalization of Liebeck [4, Corollary 4.4].

Lemma 2.2. Let $W$ be an extension of the abelian group $X$ by the group $B$ and let $f \in X$ and $b \in T$. Then

$$
(f, r b)=\prod_{i=0}^{r} f_{i}^{k(i)}=\prod_{i=0}^{r} f^{k(i)}\left(f^{k(i)}, b^{i}\right) \quad \text { where } k(i)=(-1)^{r+i}\left(\begin{array}{l}
r \\
i
\end{array}\right) .
$$

Corollary 2.3. If $W, f$, and $b$ are as in Lemma 2.2, then

$$
\begin{aligned}
\left(f, p^{j} b\right) & =\left(f, b^{p^{j}}\right) \prod_{i=1}^{p^{j}-1} f^{p t(i)}\left(f^{p t(i)}, b^{i}\right), \quad \text { podd }, \\
& =f^{2}\left(f, b^{\left.2^{j}\right)} \prod_{i=1}^{2^{j-1}} f^{2 t(i)}\left(f^{2 t(i)}, b^{i}\right), \quad p=2,\right.
\end{aligned}
$$

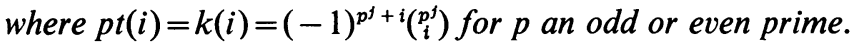

THEOREM 2.4. If $f \in X$ and $b \in T$ are as in Lemma 2.2 and if $f$ has order dividing $p^{k}$, then

(i) $(f, r b)=1$ for $r \geqq p^{k}+n(p-1) p^{k-1}$, and

(ii) $(f, r b)$ has order dividing $p^{t}$ for $t \leqq n$ and $r \geqq p^{k}+(n-t)(p-1) p^{k-1}$.

3. The lower central series of certain semidirect products. Lemma 3.1 gives a generalization of [7, Lemma 4.1] and is easily verified using the definition of commutator elements and the identities of (2.1).

LeMma 3.1. Let $W$ be an extension of the abelian group $X$ by the group $B$. Let $g, g_{i} \in W, x, x_{0}, x_{i} \in X$ and $w_{i}=g_{i} x_{i}$ for $i=1, \ldots, s$. Then

(i) $(g, x)=\left(x^{-1}, g\right)$,

(ii) $\left(x_{0}, g x\right)=\left(x_{0}, g\right)$ and $\left(x x_{0}, g\right)=(x, g)\left(x_{0}, g\right)$, and

(iii) $\left(x x_{0}, w_{1}, \ldots, w_{s}\right)=\left(x, g_{1}, \ldots, g_{s}\right)\left(x_{0}, g_{1}, \ldots, g_{s}\right)$.

Lemma 3.2. If $W$ is an extension of the abelian group $X$ by the group $B, T$ is a transversal of $X$ in $W$, and $t_{1}, t_{2} \in T$ and $x_{1}, x_{2} \in X$, then $\left(t_{1} x_{1}, t_{2} x_{2}\right) \in W_{(2)}$ has the expansion

$$
\left(t_{1}, t_{2}\right)\left(x_{2}^{-1}, t_{1}\right)\left(x_{2}^{-1}, t_{1},\left(t_{1}, t_{2}\right)\right)\left(x_{2}^{-1},\left(t_{1}, t_{2}\right)\right)\left(x_{1}^{-1},\left(t_{1}, t_{2}\right)\right)\left(x_{1}, t_{2}\right) .
$$


Proof. Expand by the identities of (2.1) and apply Lemma 3.1.

THEOREM 3.3. If $W$ is a semidirect product of the abelian group $X$ by the group $B$, then for $t \geqq 2$

$$
W_{(t)}=B_{(t)} \prod_{k=1}^{t-1}\left(X, B_{(k)},(t-1-k) B\right) .
$$

Proof. Lemma 3.2 gives the result for $t=2$ immediately. Assume $W_{(t)}=$ $B_{(t)} \prod_{k=1}^{t-1}\left(X, B_{(k)},(t-1-k) B\right)$ for $t \geqq 2$ and consider $W_{(t+1)}=\left(W_{(t)}, W\right)$. If $b_{(t)} \in B_{(t)}$ and $c \in \prod_{k=1}^{t-1}\left(X, B_{(k)},(t-1-k) B\right)$, then for $b x \in W$,

$$
\left(b_{(t)} c, b x\right)=\left(b_{(t)}, b\right)\left(x^{-1}, b_{(t)}\right)\left(x^{-1}, b_{(t)},\left(b_{(t)}, b\right)\right)\left(x^{-1},\left(b_{(t)}, b\right)\right)\left(c^{-1},\left(b_{(t)}, b\right)\right)(c, b) .
$$

But then $\left(b_{(t)}, b\right) \in B_{(t+1)}$ and hence the first four factors of $\left(b_{(t)} c, b x\right)$ from $X$ are in $\left(X, B_{(t)}\right)$ since $c^{-1} \in X$. Further $(c, b) \in \prod_{k=1}^{t-1}\left(X, B_{(k)},(t-1-k) B, B\right)$ by Lemma 3.1(ii), and hence

$$
\left(b_{(t)} c, b x\right) \in B_{(t+1)} \prod_{k=1}^{(t+1)-1}\left(X, B_{(k)},(t+1-1-k) B\right) .
$$

COROLLARY 3.4. If $W$ is a semidirect product of an abelian group $X$ by a nilpotent group $B$ of class $L$ and $t \geqq L+1$, then

$$
W_{(t)}=\prod_{k=1}^{L}\left(X, B_{(k)},(t-1-k) B\right) .
$$

The next corollary gives an explicit characterization of the groups which are the intersection of the terms of the lower central series of the standard wreath product with its base group denoted by $D_{r}$ in Meldrum [5, Lemma 2.4].

Corollary 3.5. Let $W=A$ Wr $B$ be the standard wreath product of the abelian group $A$ by the group $B$. Let $D=A^{B}$ denote the base group. Then $D_{r}=W_{r} \cap D$ $=\prod_{k=1}^{r-1}\left(D, B_{(k)},(r-1-k) B\right)$.

4. An upper bound for the nilpotency class of certain semidirect products. In this section a new upper bound for the class of a semidirect product of an abelian p-group of bounded exponent by a finite p-group is calculated. Definition 4.2 establishes the parameters of the finite $p$-group that are to be used in calculating this bound.

LeMma 4.1. Let $B$ be a finite nilpotent group and let $\Lambda: B_{0}<B_{1}<\cdots<B_{t}=B$ be any central series of $B$. Then $B_{i} / B_{i-1} \cong C_{m(i, 1)} \times \cdots \times C_{m(i, r(i))}$ where $C_{m(i, j)}$ denotes a cyclic group of order $m(i, j)$ and $m(i, j+1)$ divides $m(i, j) . r(i)$ denotes the rank of $B_{i} / B_{i-1}$. If $\left\{b_{i, 1}, \ldots, b_{i, r(i)}\right\}$ is an independent set of generators of $B_{i}$ modulo $B_{i-1}$ for $i=1, \ldots, t$, then each element $b \in B$ has a unique expression of the form

$$
b=\prod_{i=1}^{t}\left(\prod_{j=1}^{r(i)}\left(b_{i, j}\right)^{\alpha(i, j)}\right), \quad 0 \leqq \alpha(i, j)<m(i, j) .
$$


Proof. Straightforward induction on $t$ and the Fundamental Theorem for Finite Abelian Groups.

Definition 4.2. If $B$ is a finite nilpotent group, $\Lambda$ is a central series of length $t$ of $B$, and $\left\{b_{i, 1}, \ldots, b_{i, r(i)}\right\}, i=1, \ldots, t$, is as described in Lemma 4.1 , then $R(\Lambda)=$ $\bigcup_{i=1}^{t}\left\{b_{i, 1}, \ldots, b_{i, r(i)}\right\}$ is called a representation set for $B$ with respect to $\Lambda$. If $B$ is a finite $p$-group, which is now assumed unless specifically stated otherwise, then the factor groups of $\Lambda$ are internal direct products of cyclic subgroups of descending prime power order. If

$$
B_{i} / B_{\imath-1} \cong C_{p(i, 1)} \times \cdots \times C_{p(i, r(i))}
$$

where the $p(i, j)$ 's indicate the descending powers of the specified prime number $p$, then we make the following definitions dependent upon $\Lambda$ :

$$
\lambda_{i}=\sum_{j=1}^{r(i)}(p(i, j)-1), \quad i=1, \ldots, t,
$$

and

$$
\Lambda\left(X_{1}, \ldots, X_{s}\right)=\sum_{m=1}^{s-1}\left(\prod_{i=m+1}^{s} p(i, 1)\right) X_{m}+X_{s}, \quad 1 \leqq s \leqq t .
$$

The multivariable linear polynomials $\Lambda\left(X_{1}, \ldots, X_{s}\right)$ are dependent upon the exponents of the factor groups of $\Lambda$ and the $\lambda_{i}$ are dependent upon the cycle structure of the respective factor groups of $\Lambda$.

Definition 4.3. Let $W$ be a semidirect product of the abelian group $X$ by the finite nilpotent group $B$. If $R(\Lambda)$ is a representation set of $B$ with respect to the central series $\Lambda$ of $B$, then the commutator $c=\left(f, d_{1}, \ldots, d_{s}\right), f \in X$ and $d_{1}, \ldots, d_{s} \in R(\Lambda)$ is called a special commutator (s.c.) of $W$ with respect to $R(\Lambda)$. If $c$ is a special commutator of $W$ with at least $p\left(i_{0}, j_{0}\right)$ consecutive entries of the element $b_{i_{0}, j_{0}}$ appearing as the final entries of $c$, then $c\left(i_{0}, j_{0}\right)$ denotes the special commutator formed from $c$ by deleting the last $p\left(i_{0}, j_{0}\right)$ entries of $c$.

Definition 4.4. Let $W, X, B$ and $R(\Lambda)$ be as in Definition 4.3 where $\Lambda$ has length $L$. If $f \in X$ and $b_{1}, \ldots, b_{q} \in B$, then $c=\left(f, b_{1}, \ldots, b_{q}\right) \in W_{(q+1)}$ is said to satisfy the $L$-tuple of nonnegative integers $\left(a_{1}, \ldots, a_{L}\right)$ if for each $s=1, \ldots, L$ there is at least $\sum_{i=1}^{s} a_{i}$ entries in $c$ from the $s$ th term of $\Lambda$. For $1 \leqq m \leqq L, c$ satisfies $\left(a_{1}, \ldots, a_{m}\right)$ if $c$ satisfies $\left(a_{1}, \ldots, a_{m}, 0, \ldots, 0\right)$.

The next lemma, in view of Theorem 3.3, allows one to calculate an upper bound on the class of $W$ by considering certain special commutators.

Lemma 4.5. Let $W, X, B$, and $R(\Lambda)$ be as in Definition 4.3. If $f \in X$ and $b_{1}, \ldots, b_{q} \in B$ such that $c=\left(f, b_{1}, \ldots, b_{q}\right)$ satisfies $\left(a_{1}, \ldots, a_{m}\right)$, then $c$ is expressible as a product of special commutators with respect to $R(\Lambda)$ such that each has first entry $f \in X$ and satisfies $\left(a_{1}, \ldots, a_{m}\right)$.

Proof. The proof is accomplished by first expressing each $b_{i}$ in terms of its representation with respect to $R(\Lambda)$ and then applying the identities of (2.1) and Lemma 3.1. 
The following lemma gives an equation for rearranging the entries from $B$ in special commutators and facilitates the commutator calculations to follow.

LeMma 4.6. Let $W$ be a semidirect product of the abelian group $X$ by the group $B$ with $f \in X$ and $b_{1}, b_{2} \in B$. Then if $c=\left(b_{1}^{-1}, b_{2}^{-1}\right)$,

$$
\left(f, b_{1}, b_{2}\right)=(f, c)\left(f, c, b_{1}\right)\left(f, c, b_{2}\right)\left(f, c, b_{2}, b_{1}\right)\left(f, b_{2}, b_{1}\right) .
$$

Proof. If $c=\left(b_{1}^{-1}, b_{2}^{-1}\right)$, then $b_{1} b_{2}=c b_{2} b_{1}$. By the identities of (2.1) and Lemma 3.1

$$
\left(f, b_{1} b_{2}\right)=\left(f, b_{2}\right)\left(f, b_{1}\right)\left(f, b_{1}, b_{2}\right)
$$

and

$$
\left(f, c b_{2} b_{1}\right)=\left(f, b_{1}\right)\left(f, b_{2}\right)\left(f, b_{2}, b_{1}\right)(f, c)\left(f, c, b_{2}\right)\left(f, c, b_{1}\right)\left(f, c, b_{2}, b_{1}\right) .
$$

Therefore, since all factors are in $X \leqq W$, the factors commute and the result follows.

LEMMA 4.7. Let $B$ be a finite p-group with central series $\Lambda$ and let $\Lambda\left(X_{1}, \ldots, X_{s}\right)$, $1 \leqq s \leqq L$, be the polynomial functions defined in (4.2). If $\left(a_{1}, \ldots, a_{s}\right)$ is any tuple of integers, then

$$
\Lambda\left(a_{1}, \ldots, a_{j(1)}+1, \ldots, a_{j(2)}-1, \ldots, a_{j(3)}-1, \ldots, a_{s}\right) \geqq \Lambda\left(a_{1}, \ldots, a_{s}\right)
$$

for $j(1)<j(2) \leqq j(3)\left(\right.$ if $j(2)=j(3)$, replace $a_{j(2)}-1$ by $\left.a_{j(2)}-2\right)$.

\section{Proof.}

$$
\begin{aligned}
& \Lambda\left(a_{1}, \ldots, a_{j(1)}+1, \ldots, a_{j(2)}-1, \ldots, a_{j(3)}-1, \ldots, a_{s}\right) \\
& \quad=\Lambda\left(a_{1}, \ldots, a_{s}\right)+\prod_{i=j(1)+1}^{s} p(i, 1)-\left(\prod_{i=j(2)+1}^{s} p(i, 1)+\prod_{i=j(3)+1}^{s} p(i, 1)\right) .
\end{aligned}
$$

Since $j(1)<j(2) \leqq j(3)$ and $p(j(1)+1,1) \geqq 2$,

$$
\begin{aligned}
\prod_{i=j(1)+1}^{s} p(i, 1)-\left(\prod_{i=j(2)+1}^{s} p(i, 1)+\right. & \left.\prod_{i=j(3)+1}^{s} p(i, 1)\right) \\
& \geqq \prod_{i=j(1)+1}^{s} p(i, 1)-2 \prod_{i=j(2)+1}^{s} p(i, 1) \geqq 0 .
\end{aligned}
$$

The last two lemmas together with Lemma 3.1 and Lemma 4.5 establish the following theorem.

THEOREM 4.8. If $c=\left(f, d_{1}, \ldots, d_{i}, d_{i+1}, \ldots, d_{q}\right), d_{i} \in B_{s}$, is a s.c. in $W$ satisfying $\left(a_{1}, \ldots, a_{s}\right), 1 \leqq s \leqq L$, then $c=\left(f, d_{1}, \ldots, d_{i+1}, d_{i}, \ldots, d_{q}\right)$ times a product of s.c.'s having $f$ as the initial entry and satisfying

$$
\begin{array}{ll}
\left(a_{1}, \ldots, a_{t}+1, \ldots, a_{s-k}-1, \ldots, a_{s}-1\right) & \text { if } d_{i+1} \in B_{s-k} \\
\left(a_{1}, \ldots, a_{t}+1, \ldots, a_{s}-2\right) & \text { if } d_{i+1} \in B_{s} \\
\left(a_{1}, \ldots, a_{t}, \ldots, a_{s-1}+1, a_{s}-1\right) & \text { if } d_{i}+1 \in B_{s+k} .
\end{array}
$$


Furthermore, if $\left(a_{1}^{*}, \ldots, a_{s}^{*}\right)$ is any one of the s-tuples of (4.8.1), then

$$
\begin{aligned}
& \Lambda\left(a_{1}^{*}, \ldots, a_{s}^{*}\right) \geqq \Lambda\left(a_{1}, \ldots, a_{s}\right) \text { and } \\
& \Lambda\left(a_{1}^{*}, \ldots, a_{s-1}^{*}\right)>\Lambda\left(a_{1}, \ldots, a_{s-1}\right) .
\end{aligned}
$$

LEMMA 4.9. Let $W$ be a semidirect product of an abelian group $X$ by a finite $p$ group $B$ with central series $\Lambda$. If $f \in X$ has order dividing $p^{n+1}$ and $b_{1}, \ldots, b_{m}$ are elements of $B$ such that the commutator $c=\left(f, b_{1}, \ldots, b_{m}\right)$ satisfies

$$
\left(\lambda_{1}+(n-t)(p-1) p(1,1) p^{-1}+1,0, \ldots, 0\right), \quad 0 \leqq t \leqq n,
$$

then $c$ is a product of elements of $X$ of orders dividing $p^{t}$.

Proof. By Lemma 4.6, since $B_{1} \subseteq Z(B), c$ may be expressed in the form

$$
\left(f, k(1) b_{1,1}, \ldots, k(r(1)) b_{1, r(1)}, d_{1}, \ldots, d_{q}\right)
$$

with $\sum_{i=1}^{r(1)} k(i) \geqq \lambda_{1}+(n-t)(p-1) p(1,1) p^{-1}+1$. Thus, by Lemma 3.1, the proof will be complete if we show the special commutator

$$
c^{*}=\left(f, k(1) b_{1,1}, \ldots, k(r(1)) b_{1, r(1)}\right)
$$

has order dividing $p^{t}$ whenever

$$
\sum_{i=1}^{u} k(j(i)) \geqq \sum_{i=1}^{u}(p(1, j(i))-1)+(n-t)(p-1) p(1,1) p^{-1}+1
$$

for any subset $\{j(1), \ldots, j(u)\}$ of $\{1, \ldots, r(1)\}$. The procedure is by induction on $n$ as follows.

If $n=0$, then some $b_{1, j\left(i_{0}\right)}, 1 \leqq i_{0} \leqq u$, must appear as an entry in $c^{*}$ at least $p\left(1, j\left(i_{0}\right)\right)$ times and by Lemma 4.6 all entries equal $b_{1, j\left(i_{0}\right)}$ may be assumed to follow $f$ in the commutator $c^{*}$. Then by Corollary $2.3,\left(f, p\left(1, j\left(i_{0}\right)\right) b_{1, j\left(i_{0}\right)}\right)=1$ and hence it follows that $c^{*}=1$. This establishes the result for $n=0$ since $0 \leqq t \leqq n$.

Assume the result for all commutator elements $c$ having $f \in X$ of order dividing $p^{s+1}, s \leqq n$, whenever

$$
\sum_{i=1}^{u} k(j(i)) \geqq \sum_{i=1}^{u}(p(1, j(i))-1)+(s-t)(p-1) p(1,1) p^{-1}+1 .
$$

Now let $c$ be a special commutator element with $f \in X$ of order dividing $p^{n+2}$ and with

$$
\sum_{i=1}^{u} k(j(i)) \geqq \sum_{i=1}^{u}(p(1, j(i))-1)+(n+1-t)(p-1) p(1,1) p^{-1}+1
$$

for some subset $\{j(1), \ldots, j(u)\} \subseteq\{1, \ldots, r(1)\}$. Again, some entry $b_{1, j\left(i_{0}\right)}$ of $c^{*}=$ $\left(f, k(1) b_{1,1}, \ldots, k(r(1)) b_{1, r(1)}\right)$ must occur as an entry at least $p\left(1, j\left(i_{0}\right)\right)$ times and we choose $t\left(i_{0}\right)$ such that

$$
\begin{aligned}
p\left(1, j\left(i_{0}\right)\right)-1+\left(t\left(i_{0}\right)-1\right)(p-1) p( & \left.1, j\left(i_{0}\right)\right) p^{-1}<k\left(j\left(i_{0}\right)\right) \\
& \leqq p\left(1, j\left(i_{0}\right)\right)-1+t\left(i_{0}\right)(p-1) p\left(1, j\left(i_{0}\right)\right) p^{-1} .
\end{aligned}
$$


By Lemma 4.6,

$$
c^{*}=\left(f, k\left(j\left(i_{0}\right)\right) b_{1, j\left(i_{0}\right)}, k^{\prime}(1) b_{1,1}, \ldots, k^{\prime}(r(1)) b_{1, r(1)}\right)
$$

where $k^{\prime}(i)=k(i), i \neq j\left(i_{0}\right)$, and $k^{\prime}\left(j\left(i_{0}\right)\right)=0$. Then, by Theorem $2.4,\left(f, k\left(j\left(i_{0}\right)\right) b_{1, j\left(i_{0}\right)}\right)$ is an element of $X$ of order dividing $p^{(n+2)-t\left(i_{0}\right)}$. Since $p(1,1) \geqq p\left(1, j\left(i_{0}\right)\right)$, $\sum_{i=1}^{u-1} k^{\prime}\left(j^{\prime}(i)\right), j^{\prime}(i)=j(i)$ for $1 \leqq i<i_{0}$ and $j^{\prime}(i)=j(i+1)$ for $i_{0}<i \leqq u-1$, is greater than or equal to $\sum_{i=1}^{u-1}\left(p\left(1, j^{\prime}(i)\right)-1\right)+\left(\left(n+1-t\left(i_{0}\right)\right)-t\right)(p-1) p(1,1) p^{-1}+1$. Therefore the result follows by the induction hypotheses since $t\left(i_{0}\right) \geqq 1$.

THeOREM 4.10. Let $W, X, B, \Lambda$, and $f$ be as given in Lemma 4.9. If $b_{1}, \ldots, b_{m}$ are elements of $B$ and $c=\left(f, b_{1}, \ldots, b_{m}\right)$ satisfies $\left(a_{1}, \ldots, a_{s}\right), 1 \leqq s \leqq L$, such that for some $t, 0 \leqq t \leqq n$,

$$
\Lambda\left(a_{1}, \ldots, a_{s}\right) \geqq \Lambda\left(\lambda_{1}, \ldots, \lambda_{s}\right)+(n-t)(p-1) p(1,1) p^{-1} \prod_{i=2}^{s} p(i, 1)+1,
$$

then $c$ is a product of elements of $X$ of orders dividing $p^{t}$.

Proof. The proof is by induction on $n$ and $s$. The result is first verified for all $s$ when $n=0$, then assuming the theorem for a given $n \geqq 0$ and all $s$, the result is verified for $n+1$ and $s, 1 \leqq s \leqq L$.

For $n=0, t=0$, and the conclusion is equivalent to showing each such commutator is 1 . For $s=1$, Lemma 4.9 gives the result and we assume $c=1$ for all $c$ satisfying the conditions of the hypothesis for $n=0$ and a given $s \geqq 1$. Then, if $c$ satisfies the hypothesis for $s+1$, we may assume $c$ satisfies

$$
\left(a_{1}, \ldots, a_{s}, j p(s+1,1)+\lambda_{s+1}+1\right)
$$

where

$$
\Lambda\left(a_{1}, \ldots, a_{s}\right)=\Lambda\left(\lambda_{1}, \ldots, \lambda_{s}\right)-j, \quad 0 \leqq j \leqq \Lambda\left(\lambda_{1}, \ldots, \lambda_{s}\right),
$$

and has at least $\lambda_{s+1}+1$ entries from $B_{s+1}-B_{s}$ when $j=0$. The justification for $n=0$ is now completed by induction on $j$.

If $c$ satisfies (4.10.1) for $j=0$, then some $b_{s+1, j_{0}}$ must appear as an entry at least $p\left(s+1, j_{0}\right)$ times. Then by Theorem 4.8 and the induction hypothesis for $s, c$ is equal to a special commutator $c^{\prime}$ having the same entries as $c$ but having all entries equal to $b_{s+1, j_{0}}$ appearing last. Now by Corollary 2.3, since $f$ has order dividing $p$,

$$
c^{\prime}=\left(c^{\prime}\left(s+1, j_{0}\right),\left(b_{s+1, j_{0}}\right)^{p\left(s+1, j_{0}\right)}\right)
$$

Therefore $c^{\prime}$, and thus $c$, is equal to 1 since $\left(b_{s+1, j_{0}}\right)^{p\left(s+1, j_{0}\right)} \in B_{s}$. Using the inductive assumption for $j \geqq 0$ in place of the induction hypothesis for $s$ in the above argument gives the conclusion for all $j$ and thus completes the proof for $n=0$.

We now assume the theorem for all special commutators with $f$ of order dividing $p^{n+1}$ and observe that Lemma 4.9. gives the desired conclusion for special commutators having $f$ of order dividing $p^{n+2}$ and $s=1$. Thus, we assume the divisibility 
condition for all special commutators with the order of $f$ dividing $p^{n+2}$ and satisfying the conditions of the hypothesis for a given $s \geqq 1,1 \leqq s \leqq L-1$. Then, if $c$ satisfies the hypothesis for $s+1$, we may assume $c$ satisfies

$$
\left(a_{1}, \ldots, a_{s}, j p(s+1,1)+\lambda_{s+1}+1\right)
$$

where

$$
\begin{aligned}
\Lambda\left(a_{1}, \ldots, a_{s}\right) & =\Lambda\left(\lambda_{1}, \ldots, \lambda_{s}\right)+(n+1-t)(p-1) p(1,1) p^{-1} \prod_{i=2}^{s} p(i, 1)-j, \\
0 & \leqq j \leqq \Lambda\left(\lambda_{1}, \ldots, \lambda_{s}\right)+(n+1-t)(p-1) p(1,1) p^{-1} \prod_{i=2}^{s} p(i, 1),
\end{aligned}
$$

and has at least $\lambda_{s+1}+1$ entries from $B_{s+1}-B_{s}$ when $j=0$. Again we proceed by induction on $j$. If $j=0$, then some $b_{s+1, j_{0}}$ must appear at least $p\left(s+1, j_{0}\right)$ times as an entry of $c$ and by Theorem 4.8 and the induction hypothesis for $n+1$ and $s, c$ is equal to a product of elements of $X$ of orders dividing $p^{t}$ times a special commutator $c^{\prime}$ having the same entries as $c$ but having all entries equal to $b_{s+1, j_{0}}$ appearing as its final entries. Then, by Corollary 2.3 for $p$ an odd prime,

$$
c^{\prime}=\left(c^{\prime}\left(s+1, j_{0}\right),\left(b_{s+1, j_{0}}\right)^{p\left(s+1, j_{0}\right)}\right) \prod_{m=1}^{p\left(s+1, j_{0}\right)-1} c^{\prime}\left(s+1, j_{0}\right)^{p k(m)}\left(c^{\prime}\left(s+1, j_{0}\right)^{p k(m)}, b_{s+1, j_{0}}^{m}\right) .
$$

Since $\left(b_{s+1, j_{0}}\right)^{p\left(s+1, j_{0}\right)} \in B_{s}$, the first factor is a product of elements of $X$ of orders dividing $p^{t}$ by the inductive assumption for $s$. By Lemma 3.1, if $c^{\prime}\left(s+1, j_{0}\right)^{p k(m)}$ has order dividing $p^{t}$, then so does $\left(c^{\prime}\left(s+1, j_{0}\right)^{p k(m)}, b_{s+1, j_{0}}^{m}\right)$. Now $c^{\prime}\left(s+1, j_{0}\right)^{p k(m)}$, by Lemma 3.1 , is a special commutator with first entry $f^{p k(m)}$ and satisfies $\left(a_{1}, \ldots, a_{s}, \lambda_{s+1}+1-p\left(s+1, j_{0}\right)\right)$. Then since $f^{p k(m)}$ has order dividing $p^{n+1}$, we are done if $t=n+1$. Otherwise, $t \leqq n$ and

$$
\begin{aligned}
\Lambda\left(a_{1}, \ldots,\right. & \left.a_{s}, \lambda_{s+1}+1-p\left(s+1, j_{0}\right)\right) \\
= & \Lambda\left(a_{1}, \ldots, a_{s}\right) p(s+1,1)+\lambda_{s+1}+1-p\left(s+1, j_{0}\right) \\
= & {\left[\Lambda\left(\lambda_{1}, \ldots, \lambda_{s}\right)+(n+1-t)(p-1) p(1,1) p^{-1} \prod_{i=2}^{s} p(i, 1)\right] p(s+1,1) } \\
& +\lambda_{s+1}+1-p\left(s+1, j_{0}\right) \\
\geqq & \Lambda\left(\lambda_{1}, \ldots, \lambda_{s+1}\right)+(n-t)(p-1) p(1,1) p^{-1} \prod_{i=2}^{s+1} p(i, 1)+1 .
\end{aligned}
$$

Therefore, by the induction hypothesis for $n$ and any $s, 1 \leqq s \leqq L, c^{\prime}\left(s+1, j_{0}\right)^{p k(m)}$ is a product of elements of $X$ of orders dividing $p^{t}$. The proof by induction on $j$ follows by replacing the inductive assumption for $n+1$ and $s$ by the inductive assumption for $j \geqq 0$ in the statements of the last argument. Also, the case $p=2$ requires no special attention because of the similarity of the expressions in Corollary 2.3 and its use in the above argument. 
THEOREM 4.11. If $W$ is a semidirect product of an abelian group $X$ of exponent $p^{n+1}$ by a finite p-group $B$ of class $L$ and $\Lambda: B_{0}<B_{1}<\cdots<B_{L}=G_{(L+1)}<G_{(L)}<\cdots$ $<G_{(1)}$ is the lower central series of $B$, then

$$
\mathrm{Cl}(W) \leqq \Lambda\left(\lambda_{1}, \ldots, \lambda_{L}\right)+n(p-1) p(1,1) p^{-1} \prod_{i=2}^{L} p(i, 1)+1
$$

where $\lambda_{i}, i=1, \ldots, L$, and $\Lambda\left(X_{1}, \ldots, X_{L}\right)$ are as defined by Definition 4.2.

Proof. Let $q=\Lambda\left(\lambda_{1}, \ldots, \lambda_{L}\right)+n(p-1) p(1,1) p^{-1} \prod_{i=2}^{L} p(i, 1)+1$. Then by Corollary 3.4 there is a set of generators of $W_{(q+1)}$ such that each generator satisfies $(0, \ldots, 1,0, \ldots, q-k)$, an $L$-tuple with 1 in the $k$ th coordinate, $1 \leqq k \leqq L$. Thus each generator must be the identity element by Theorem 4.10 since

$$
\begin{aligned}
\Lambda(0, \ldots, 1,0, \ldots, q-k) & =\prod_{i=L-k+2}^{L} p(i, 1)+q-k \\
& \geqq p^{L-(L-k+2)+1}+q-k=p^{k-1}+q-k \geqq q \text { for all } k .
\end{aligned}
$$

The following example illustrates the improvement of Theorem 4.11 over the upper bound of Scruton [7, Lemma 4.6] which was calculated for wreath products a special type of semidirect product.

EXAMPLE 4.12. Let $A$ be an abelian group of exponent $p^{n+1}$ and $B$ the relatively free nilpotent group of class 3 , generated by three elements, and of exponent $p$. Then (see [6, Lemma 31.61])

$$
\begin{aligned}
& B_{(1)} / B_{(2)} \cong C_{p} \times C_{p} \times C_{p}, \\
& B_{(2)} / B_{(3)} \cong C_{p} \times C_{p} \times C_{p}, \text { and } \\
& B_{(3)} / B_{(4)} \cong C_{p} \times C_{p} \times C_{p} \times C_{p} \times C_{p} \times C_{p} \times C_{p} \times C_{p} .
\end{aligned}
$$

Let $W$ be the standard wreath product of $A$ by $B$ (see [1], [4], or [7]). Then $W$ is a semidirect product of an abelian group of exponent $p^{n+1}$ by $B$ and hence by Theorem 4.11

$$
\begin{aligned}
\mathrm{Cl}(W) & \leqq \Lambda(8(p-1), 3(p-1), 3(p-1))+n(p-1) p^{2}+1 \\
& =\left((n+8) p^{2}+3 p+3\right)(p-1)+1 .
\end{aligned}
$$

The upper bound given by Scruton [7, Lemma 4.6] would be $n p^{12}(2 p-1)$ since the order of $B$ is $p^{14}$.

The author expresses his gratitude to Professor Paul M. Weichsel, his Ph.D. Thesis Advisor, for having suggested this topic for research study.

\section{REFERENCES}

1. G. Baumslag, Wreath products and p-groups, Proc. Cambridge Philos. Soc. 55 (1959), 224-231. MR 21 \#4179.

2. Philip Hall, Nilpotent groups, Canadian Mathematical Congress, Summer Seminar, University of Alberta, 1957. 
3. L. Kaloujnine and Marc Krasner, Produit complet des groupes de permutations et le problème d'extension des groupes. III, Acta Sci. Math. (Szeged) 14 (1951), 44-62.

4. Hans Liebeck, Concerning nilpotent wreath products, Proc. Cambridge Philos. Soc. 58 (1962), 443-451. MR 25 \#3087.

5. J. D. P. Meldrum, Central series in wreath products, Proc. Cambridge Philos. Soc. 63 (1967), 551-567. MR 35 \#4309.

6. Hanna Neumann, Varieties of groups, Ergebnisse der Mathematik und ihrer Grenzgebiete, Band 37, Springer-Verlag, New York, 1967. MR 35 \#6734.

7. Teresa Scruton, Bounds for the class of nilpotent wreath products, Proc. Cambridge Philos. Soc. 62 (1966), 165-169. MR 32 \#5721.

\section{WESTERN ILLINOIS UNIVERSITY,}

MACOMB, ILLINOIS 61455 